Journal of Research in Interprofessional

Practice and

Education

Vol. 2.1

Editorial

July, 2011
Journal of Research in Interprofessional Practice and Education (JRIPE)

Vol. 2.1

(C) 2011

Corresponding author: Paula Rowland

Email: !paula.rowland@gmail.comi

\section{Making the Familiar Extraordinary: Using a Communication Perspective to Explore Team-Based Simulation as Part of Interprofessional Education}

\author{
Paula Rowland, HBSc, BHSc (OT), MA
}

\begin{abstract}
Background: This short report describes the qualitative analysis of a simulation exercise that was part of a larger interprofessional education curriculum designed to promote interprofessional collaboration within an acute care hospital. The purpose of this study was to explore the utility of a communication perspective as an analytic tool to support the intended learning outcomes of the interprofessional education initiative.

Methods and Findings: The Coordinated Management of Meaning model was used as an exemplar of a pragmatic communication perspective.

Conclusion: This study supports the need for scholars, practitioners, and educators to critically reflect on what meaning we are making within our interprofessional education initiatives. The Coordinated Management of Meaning model provides a useful analytic frame to shape that reflection.

Keywords: Interprofessional education; Simulation; Coordinated management of meaning model
\end{abstract}

\section{Introduction}

In a recent editorial, Reeves [1] called upon scholars and practitioners to problematize elements of our work within interprofessional collaboration and education. The concept of problematization originates within critical theory. To problematize an idea is an invitation to become intentionally critical of the assumptions that have become normalized in practice and research. This critical analysis allows scholars and practitioners to recognize the constraints assumptions place on our collective thinking. In this sense, criticality is not intended to devalue an idea. Instead, the process of reflective critical analysis allows us to understand phenomena within a new light, perhaps revealing new opportunities within historically entrenched challenges. It is within this critical analysis that creativity is released and new knowledge is generated.

This short report describes the qualitative analysis of a simulation exercise that was part of a larger interprofessional education curriculum designed to promote interprofessional collaboration within an acute care hospital. The purpose of this study was to explore the utility of the Coordinated Management of Meaning model as an analytic tool to support the intended learning outcomes of the interprofessional education initiative. The Coordinated Management of Meaning model is a pragmatic model based on a communication perspective. This report describes the interprofessional education project, the Coordinated Management of Meaning model that was used for analysis, and key findings of the analysis. 
Making the Familiar Extraordinary

Rowland

Journal of Research in Interprofessional Practice and Education

Vol. 2.1

July, 2011

\section{The Interprofessional Education Project}

In response to a recent surge of interest to create, support, and sustain interprofessional collaborative practice, a community based teaching hospital designed and implemented an interprofessional education program in an acute medical unit. This project was partially funded by the Health Force Ontario initiative, a joint venture between Ontario's Ministry of Health and Long Term Care, and the Ministry of Training, Colleges, and Universities. For the purposes of this project, interprofessional collaboration was defined as practice where "multiple health workers from different professional backgrounds provide comprehensive services by working together synergistically along with patients, their families, carers, and communities to deliver the highest quality of care across settings" [2]. The project created opportunities for healthcare workers within an acute care team to learn about, from, and with each other in order to enable effective interprofessional collaboration.

The project included a mix of didactic and experiential learning, divided among three modules, delivered over an 18-month time frame. The first module involved workshop-style learning focused on communication strategies, emotional intelligence, scopes of practice, and conflict resolution. The second module used standardized patients to illustrate the principles of interprofessional collaboration. As part of the simulation, participants engaged in an interprofessional conversation to relay their findings from the discipline-specific, patient-based simulation that had occurred earlier in the day. The intention of the simulated conversation was to negotiate an interprofessional care plan. This element of the simulation emulated the team conversation typically called "interprofessional rounds," an important and familiar element of the team's work within the acute-care setting. Participants were video taped as part of the simulation exercise, providing the opportunity for a facilitator-led debriefing augmented by the use of the video. The final module was also in a workshop format, leading participants through a process to design and implement a change within their unit that would support collaborative practice.

Staff enrolled as participants in the entire project included one physiotherapist (PT), two registered nurses (RN), four registered practical nurses (RPN), two social workers (SW), one registered dietitian (RD), one occupational therapist (OT), one occupational therapy/physiotherapy aide (OTA/PTA), one speech-language pathologist (SLP), and two personal care aides (PCA).

This exploratory research study focused on the simulation aspect of the project, using the video that was created of the simulated interprofessional rounds conversation. The Coordinated Management of Meaning (CMM) was the model used to guide the qualitative analysis of the video from a communication perspective. The CMM Model is described in the next section.

\section{The Coordinated Management of Meaning Model}

The Coordinated Management of Meaning model was conceived by two social scientists, Barnett Pearce and Vernon Cronen. A simplified history of the use of CMM suggests that it began as an interpretive social science in the 1970s, developed a critical edge in the 1980s, and currently expresses itself as a practical theory [3]. 
Making the Familiar Extraordinary

Rowland

Journal of Research in Interprofessional Practice and Education

Vol. 2.1

July, 2011
The CMM model is concerned with two aspects of communication: the coordination of actions and the management of meaning between people. The term coordination refers to the ways people put their communication turns together when they are in conversation. Coordination serves as a sensitizing concept within the model, asking us to look at structural aspects of the conversation, such as who spoke first or who speaks most frequently. Looking at the coordination of communication allows us to notice patterns. As a familiar pattern in healthcare, consider how many requests are preceded with the phrase "do you have a minute?" For those members who are familiar with the pattern, people will recognize that a "minute" is very rarely a literal minute, and that phrase is often followed by some kind of request. As another example, there may be a pattern within interprofessional rounds such that a particular person or role always initiates the discussion of a patient case. Part of that pattern of coordination may also include the completion of the discussion, which may only happen once a particular person or role provides their input.

Looking at the coordination of conversational turns provides two important insights into communication. First, it allows us to consider communication in a way distinct from the transmission model. The transmission model of communication looks at communication as the act of sending an idea from one person to another. The emphasis is on the sender and the receiver, looking at the alignment between the intended message and the resultant interpretation. Instead of thinking about communication as the translation of an idea from one head to another, looking at coordination of turns encourages us to consider the ways communicative turns are put together and how the pattern of what is said impacts the meaning of what is created in conversation. The exact same phrase may create very different things in conversation depending on what phrases precede it.

Second, noticing communication as a contingent process rather than an act translation allows us to think differently about interventions with teams. If we think about communication solely in terms of the way meaning is translated from one person to another, our interventions focus on clarifying the intended meaning. Looking at communication as shaped by coordination allows us to think about changing patterns of communication. For example, there may be patterns of communication that are collaborative and patterns that are competitive. In this case, the intervention could involve noticing the patterns and attempting to shape conversational structures that are consistent with what the team wants to create. Structured communication tools such as checklists could be viewed as attempts to change the structure of conversation. Looking directly at the coordination of communication allows us to evaluate whether these structures are serving our intended purposes of interprofessional collaboration to promote patient care.

In the CMM model, the two faces of communication include coordinating actions and managing meaning. These two processes can be differentiated, but they are intricately linked. There can be no meaning without action and no action without meaning [4].

Using a communication perspective, I assume that human beings are always making meaning of their context. The way people make meaning of their context 
Making the Familiar Extraordinary

Rowland

Journal of Research in Interprofessional Practice and Education

\section{Journal of Research in Interprofessional Practice and Education}

depends on their own personal biographies, their particular context, and where they are placed in the conversation. The CMM model asks us to consider "what specific meanings are (people) making in given situations, how are they making those meanings, and how those meanings affect the social world that they are making" [4].

Looking at the coordination of action and the management of meaning allows us to notice what Pearce calls "critical moments." These are moments in time in which what we do changes the social worlds in which we live [4]. Critical moments are a key concept within the theory of CMM. As one example of a critical moment, Pearce [4] analyzes the transcript of an emergency call in California. In this call, a concerned driver is telling the dispatcher that there is a mattress in the middle of a busy highway. In response to the dispatcher's question about the nature of her concern, the driver relays that the mattress is a significant hazard, saying, "Well, it took me forever to get through, and people are dodging this mattress and I just wanted to, maybe ..." [4]. The dispatcher responds, “Ok. But what I am telling you, ma'am, is that the San Jose Police do not respond to the freeway. It is the Highway Patrol's jurisdiction. I'd be more than happy to give you the number if you'd like" [4]. Shortly following the conclusion of this call, a driver hit the mattress and was subsequently killed. Pearce identifies this particular snippet of the conversation as a "critical moment" where the dispatcher had several options beyond the bureaucratic response. Pearce argues that the analysis of the critical moment requires attention to both the coordination of actions within the conversation and the meanings that were created.

Critical moments are significant from a scholarly and practical perspective. Critical moments have afterlives, meaning that they stay in the memories of those involved and in the experience of those impacted. These afterlives are carried forward into new interactions, shaping how we perceive these new experiences based on what we have learned in the past [4]. The analysis of critical moments increases our ability to recognize those moments while we are in them, improving our opportunities to act wisely in the moment. It is through the shaping of critical moments that Pearce claims the CMM model contributes to better social worlds.

\section{Application of the Coordinated Management of Meaning Model}

As a practical theory, CMM invites scholars and practitioners to take a direct look at the practices of communication [4]. It is a "social technology" [5] that allows mindful inquiry into situations. As a form of inquiry, CMM follows a rough pattern of description, interpretation, critical analysis, and practice [4]. The process is not as linear as this list may suggest. Instead, there is much interfolding, interpenetrating, and cross-informing of the phases [4].

It is within this frame that CMM answers three paradigmatic questions: a) What are we making? b) How are we making it? and, c) How do we make better social worlds?

What are we making?

Pearce [4] describes a communication perspective as a worldview that looks at com- 
Making the Familiar Extraordinary

Rowland

Journal of Research in Interprofessional Practice and Education

Vol. 2.1

July, 2011

\section{Journal of Research in Interprofessional Practice and Education}

munication rather than through it, carrying the assumption that communication is both substantive and consequential. This means that language is a material object that can be studied and that varied forms of communication have differential effects [5]. In this view, our language is performative. For example, a promise does not just describe our current state. Instead, a promise suggests and creates a new action. Following this example, a communication perspective suggests that language does not just describe our social world, it creates it.

\section{How are we making it?}

The previous description of CMM is radically opposed to the traditional model of communication as a transmission. Rather than considering communication as an act of transferring ideas, wishes, or directives from one mind to another, the CMM perspective emphasizes that social worlds are created through the coordination and management of meaning between people. As a practical theory, CMM provides several tools to describe and inquire in the ways these social worlds are made.

\section{How do we make better social worlds?}

The concepts and tools within CMM help us to understand how social worlds are created. The critical edge of CMM asks us to consider how to make better social worlds. Social worlds are shaped by changing the patterns of communication, by resisting or preventing the performance of undesirable communication patterns, or by facilitating the emergence of desirable ones [4].

Using the heuristics of CMM, we can intentionally reflect upon critical moments in the patterns of communication that shape our social worlds. These critical moments present as turning points, and they can be recognized through the mindful application of the aforementioned heuristics. The challenge for the practitioner is to recognize these critical moments in real time and to act wisely upon them, thereby creating our desired social worlds. The practitioner can do this by attending to both meaning and coordination of actions in conversation.

\section{Methods}

Following the approval of the Research Ethics Board of Fielding Graduate University and the participating hospital, I requested the participants' permission to view the video of the simulation. As explained to the participants, the intention in reviewing the video was to apply the principles of CMM in an analysis of group process from a communication perspective. The study was not concerned with the clinical skill revealed in the videos, only with the coordination of actions and the management of meaning in conversation. All eight participants provided written consent to participate in this study.

\section{Participants}

All eight participants were clinicians within the community-based hospital. Professions represented included nursing (3), dietetics (1), physiotherapy (1), physiotherapy aide (1), social work (1), and speech-language pathology (1). Participants 
Making the Familiar Extraordinary

Rowland

Journal of Research in Interprofessional Practice and Education

Vol. 2.1

July, 2011

\section{Journal of Research in Interprofessional Practice and Education}

were familiar with one another and had existing working relationships within the acute medical unit of the participating hospital.

\section{Methodology}

I first viewed the entirety of the video, including the individual interactions of the practitioners with the simulated patient. Following this initial immersion, I chose a single episode to analyze in greater depth. In the simulation exercise, the facilitators deliberately applied the simulated patient's sling incorrectly. This created an opportunity for the team members to notice a mistake and address that mistake within a group discussion. I chose to analyze the dialogue that emerged when one participant initiated a conversation about the observed "mistake."

I chose this moment in time because it fit Pearce's [4] definition of a critical moment. Namely, this moment was reliant on what had preceded it, the moment would influence the actions that would be taken next, and the moment had significant consequences for the object of the conversation. In this case, the object of the conversation was patient safety. Furthermore, I was particularly interested in this critical moment as it illuminated several of the interprofessional collaboration competencies outlined in the Canadian Interprofessional Health Collaborative (CIHC) National Interprofessional Competency Framework [6].

I used several heuristics within the CMM model to guide my analysis. First, I used the serpentine model to track the conversational turns. The serpentine model is one of the heuristics of CMM and involves a detailed analysis of the transcribed dialogue. Using the serpentine model allows the researcher to follow how reality is co-constructed, how each action could have multiple meanings, and to appreciate the contingency of social acts [5].

The second heuristic I used was the hierarchy model. The hierarchy model builds on Gregory Bateson's idea of metacommunication, acknowledging that higher levels of abstraction modify lower levels [4]. The meaning of each turn is modified by the turns preceding it, but also by the episode within which it occurs [4]. The model is created by mapping the levels of context subsumed within the episode. The levels of context are arranged in a hierarchy, demonstrating which level of context has the highest meaning in the interpretation of the speech acts within that particular episode.

The hierarchy model recognizes that people live in multiple stories at any given moment. In addition to the clinical roles as part of the interprofessional team, practitioners are members of professions and are also parents, children, siblings, taxpayers, and so on. These multiple roles provide multiple perspectives by which to consider any given situation. Pearce [4] calls these perspectives the stories in which people are living. While multiple stories may be simultaneously present, they are not all equally salient in any given moment. The CMM hierarchy model functions as a tool to enable us to inquire, in any given instance, which story is the most salient and provides context for other stories we hold. 


\section{JRIPE}

127

Making the Familiar Extraordinary

Rowland

\section{Findings}

Figure 1 graphically depicts the turn-by-turn discussion involving the registered nurse (RN), social worker (SW), and physiotherapist (PT). Verbatim dialogue is captured in quotations while a summary of the discussion is depicted in italics. Note that the turns of conversation are delineated by what was said, not by who said it. So, the PT has many turns in this particular episode as she relays her observations, the information she acquired through interview, her actions, and her assessment of the patient's reaction to her actions.

Figure 1

\section{Turn by turn transcript of the communication episode between the RN, PT, and SW}

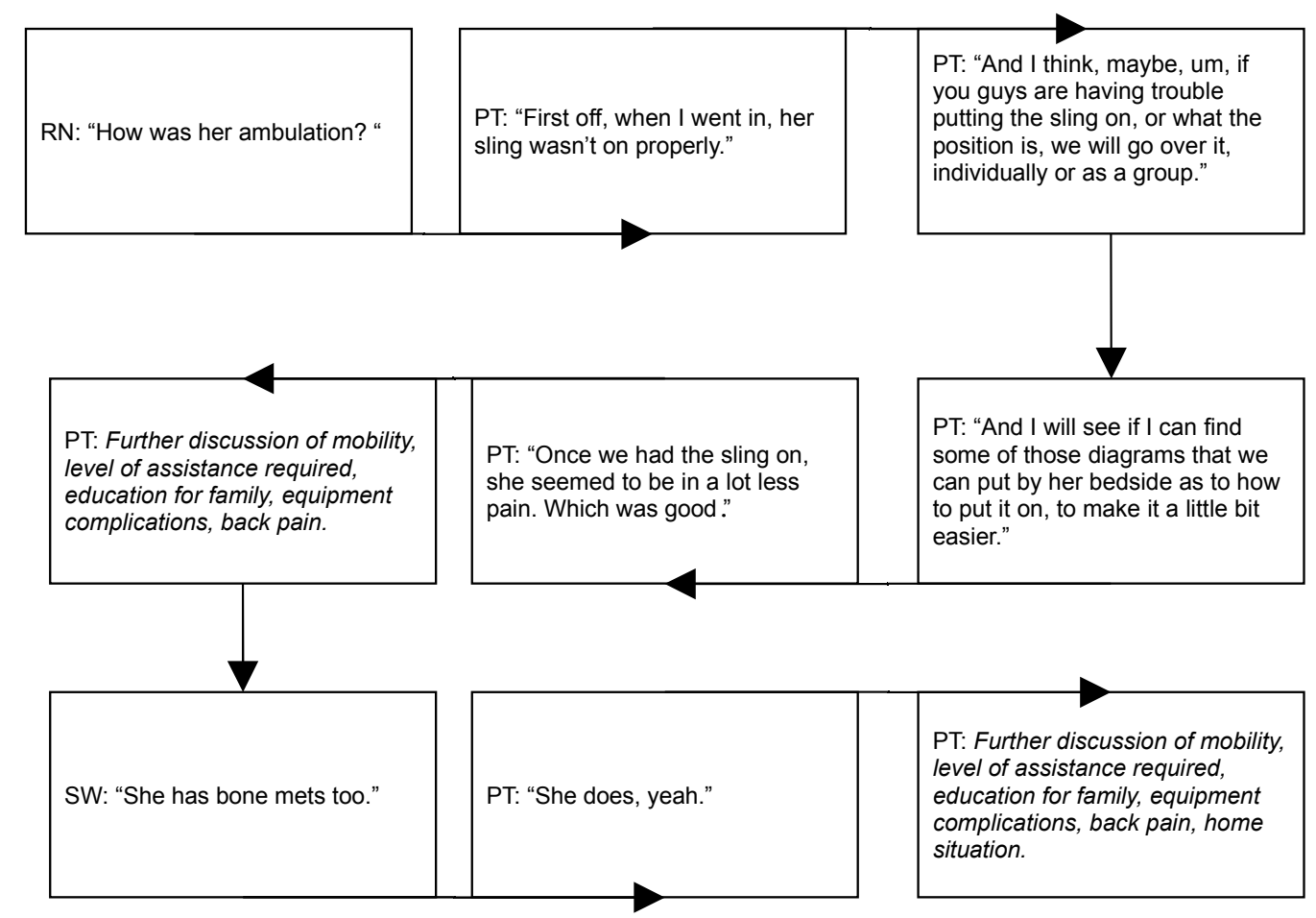

Looking at this episode with respect to the coordination of actions, I notice the PT spoke for approximately two minutes. Nearly every participant displayed this pattern of uninterrupted monologue. The way in which each professional presented their findings without interruption from other team members contributed to my interpretation of rounds as a time to present one's findings.

The PT responded to the RN's question about ambulation with a discussion of sling placement. Since it is a conversational norm to answer the question that has

Journal of Research in Interprofessional Practice and Education

Vol. 2.1

July, 2011 been posed, the PTs act of not answering the question and instead offering the unsolicited observation of the incorrect sling placement had the conversational effect of elevating the importance of her observation. However, as the PT's monologue continued, the nesting of the conversation about the sling within a range of other topics without pause for discussion positioned that element of the PTs findings as having 
Making the Familiar Extraordinary

Rowland

Journal of Research in Interprofessional Practice and Education

Vol. 2.1

July, 2011 similar importance to all her other subsequent findings. At the end of the PT's report, the conversation about the sling was not carried forward in the team's dialogue. The result is that it is unclear to me how the same mistake will be avoided in the future as the team membership shifts. As an observer, I wondered whether the team would decide to engage in in-person education or to increase the number of signs that demonstrated correct sling placement. Will either of those strategies be effective? Are there other strategies that may have more significant impact for this patient and subsequent patients? Of course, this was a simulation and there will be no carry over of activity for this simulated patient. Yet, the simulation is intended to demonstrate and reinforce the skills of interprofessional collaboration that we see as part of team practice. Therefore, I think the question of sustainability is a relevant one to consider, even within a simulation context. Presumably, we want to simulate and reinforce strategies that will have a sustainable and positive impact on patient care.

As the second stage of the analysis, a hierarchy model was created to represent the hypotheses on the contextual layers moderating the meaning of the conversation. The PT may have been experiencing a feeling of professional obligation to mention the placement of the sling related to her interpretation as "self as clinical expert." Pearce calls this a sense of "oughtness," referring both to what a person like me ought to do in a situation like this, and what the preceding turn in the conversation dictates about what I can do, must do, or should not do [4]. At any moment, we may experience different, and potentially conflicting, sources of "oughtness."

As the hierarchy model in Figure 2 graphically displays, the PT may have been holding multiple stories about who she is, and who she should be, within this context. She is simultaneously a responsible professional, a supportive team member, and a participant in an interprofessional education simulation. Each role that she is holding brings with it a different sense of oughtness. As a professional, she may feel an obligation to correct the team on the improper sling placement. As a supportive team member, she does not have any legitimate authority to instruct team members on proper sling use. As a participant in an interprofessional education simulation, she probably realizes that she is expected to manage this potential conflict in a way that demonstrates the competencies of interprofessional collaboration. The way the PT holds these different stories in context, and the importance she puts to each of these potential stories, impacts what she decides to do next. Figure 2 displays how the PT's ordering of importance of the different stories may impact her decision about what to do next.

The strategy the PT chose to resolve this potentially conflicting experience of "oughtness" is to engage the group by offering education. This offer is nested within the rest of her presentation, and it is not clear how the offer will be taken up or if her educational methods will have long-term impact outside of the membership of this particular conversation.

The hierarchy model is not intended to create an analysis of "what is truly happening here." Instead, the model creates a visual representation of multiple hypotheses that could explain the choices people have made in conversation. The veracity of these hypotheses can only be checked through dialogue with the involved person. 


\section{JRIPE}

129

Making the Familiar Extraordinary

Rowland

Journal of Research in Interprofessional Practice and Education

Vol. 2.1

July, 2011
Figure 2

Three different hypotheses about the "hierarchy of meaning" the PT
may be holding and what the most reasonable course of action would
be, depending on what context the PT deemed to be most important.

Patient advocate

Supportive team member as educator

Clinical expert as equal member of the team

Patient advocate

Clinical expert

Supportive team member

Most reasonable course of action: Create a

Most reasonable course of action: Offer an invitation to education
Simulation participant

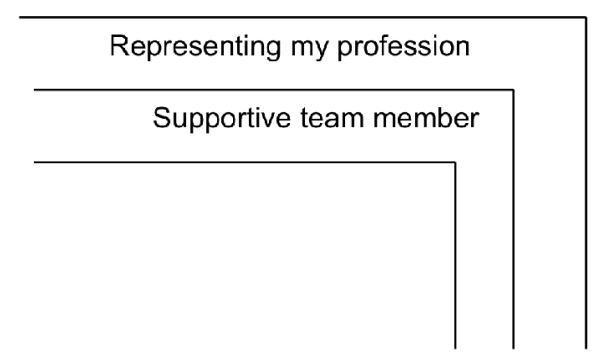

Most reasonable course of action: Structure conversation in a way that reflects the course content and anticipated expectations of the course facilitators

In team situations, the construction of these hierarchies could demonstrate how interprofessional conflicts arise. If one professional holds their role as "patient advocate" as their most important context while another team member holds their role as "supportive team member" as having primacy, the feelings of "oughtness" they each experience will be quite different. While each will feel rightly justified in their actions, the actions of their team member will seem confusing. Articulating the hierarchy of meaning that each team member is holding allows assumptions about expected behaviours to be made explicit. This can reveal sources of disagreement as being related to the meaning each team member is creating in the moment and not necessarily to an interpersonal conflict.

\section{Discussion}

This exploratory research project reveals the experience of rounds as a social accomplishment. Conceptually playing with the heuristics of CMM encouraged me 
Making the Familiar Extraordinary

Rowland

Journal of Research in Interprofessional Practice and Education

Vol. 2.1

July, 2011 to take seriously the notion of looking at communication, rather than through it. Through the reflexive application of these heuristics, different elements of the process began to seem extraordinary. How did the whole group know when they were done? If the group did not seem to be explicitly making decisions, what was the work of the group in conversation? What did the various conversational turns between professionals accomplish? This opened a whole new level of inquiry and allowed the familiar to be seen as something exceptional.

It is this ability to see familiar activities as something unusual that allows me to productively problematize aspects of interprofessional education. This problematization stimulates reflexive evaluation and critical analysis, contributing to new knowledge development at the intersection between reflexivity and critical thinking. I believe this is the kind of creativity that Reeves [1] is hoping to inspire through his editorial commentary.

This simulation exercise allowed participants an opportunity to practice a social interaction. Using CMM as a conceptual frame to guide a group reflection on the exercise would have called attention to the social accomplishments, encouraging participants to reflect upon what meaning was being made in conversation, and what was now possible following that social construction. This level of reflection would have involved participants in discovering whether their social interactions were serving the purposes they were hoping for. Simulation with guided de-briefing could become an opportunity to create new patterns of interaction, creating space to develop and practice new norms with careful and reflective attention to the ways social patterns of interaction serve our intended goals. This level of analysis brings new depth to the conversation of interprofessional education, suggesting that we need to be mindful of what stories of teams we are creating and the processes we are institutionalizing through our interprofessional education efforts.

This study is limited by the scope of research, including a short observation period and the emphasis on a limited number of communication episodes. However, this short report demonstrates that a communication perspective allows the scholar to problematize assumptions of interprofessional education, creating conceptual space for critical analysis and creative new thinking. In particular, CMM provides a collection of heuristics that creates a frame for qualitative rigor in the analysis of interprofessional education efforts. For the practitioner, CMM tools reveal turning points within conversation, thereby increasing our capacity to act wisely into critical moments. Further exploration of the academic and pragmatic offerings of a communication perspective within the field of interprofessional education and collaboration would draw upon rich traditions and insightful research from other disciplines, contributing to ongoing knowledge development within this emerging field.

\section{References}

1. Reeves, S. (2010). The need to problematize interprofessional education and practice activities. Journal of Interprofessional Care 24(4): p. 333-335.

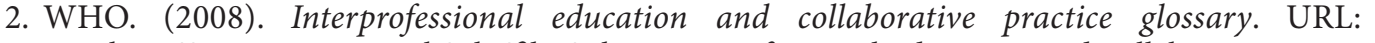

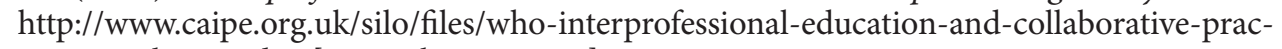
'tice-iecpglossary.docín' [December 21, 2010]. 


\section{JRIPE \\ Journal of Research in Interprofessional Practice and Education}

131

Making the Familiar Extraordinary

Rowland
3. Barge, J.K. \& Pearce, W.B. (2004). A reconnaissance of CMM research. Human Systems: The Journal of Systemic Consultation and Management 15(1): p. 13-32.

4. Pearce, W.B. (2007). Making social worlds: A communication perspective. Malden, MA: Blackwell Publishing.

5. Pearce, W.B. (2009). Investigar desde la mente adecuada [Doing research in our right minds]. In E.B. Castro, J.H. Prieto, and C.J. Herrera, (Eds.). La investigación: Aproximaciones a la construcción de Conocimiento Científico [Research:_Approaching scientific knowledge building].



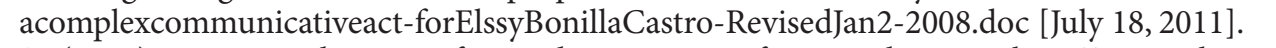

6. CIHC. (2010). A national interprofessional_ competency framework. URL: thttp://www.cihc.ca! '/files.CIHC IPCompetencies_Feb2010.pdf [December 21]. 